\title{
Effects of quantum interference in spectra of cascade spontaneous emission from multilevel systems
}

\author{
A.A. Makarov ${ }^{1,2,}{ }^{*}$, and V.I. Yudson ${ }^{1,3}$ \\ ${ }^{1}$ Institute for Spectroscopy RAS, 108840 Moscow, Troitsk, Russia \\ ${ }^{2}$ Moscow Institute of Physics and Technology, 141700 Dolgoprudny, Russia \\ ${ }^{3}$ Russian Quantum Center, 143025 Skolkovo, Russia
}

\begin{abstract}
A general expression for the spectrum of cascade spontaneous emission from an arbitrary multilevel system is presented. Effects of the quantum interference of photons emitted in different transitions are analyzed. These effects are especially essential when the transition frequencies are close. Several examples are considered: (i) Three-level system; (ii) Harmonic oscillator; (iii) System with equidistant levels and equal rates of the spontaneous decay for all the transitions; (iv) Dicke superradiance model.
\end{abstract}

The standard textbook expression (see, e.g., Ref. [1]) for the spectrum of spontaneous emission from a $(\mathrm{N}+1)$-level system with the states $|0\rangle,|1\rangle, \ldots,|N\rangle$ includes a set of Lorentzians centered at the frequencies $\omega_{N, N-1}, \ldots, \omega_{21}$, and $\omega_{10}$, with their widths $\Gamma_{N, N-1}+\Gamma_{N-1, N-2}, \ldots, \Gamma_{21}+\Gamma_{10}$, and $\Gamma_{10}$, respectively. However, if the transition frequencies are close, the Lorentzians are superimposed. This means that the correspondence of a photon to a particular transition is a priori unknown, and this is just the case of the quantum interference. Nevertheless, even for such a more complicated situation the generic formula for the spectrum exists, although its derivation is far from being routine [2]. This exact formula is given by

$$
S(\omega)=\frac{1}{2 \pi i} \sum_{l=1}^{N} \frac{1}{\Gamma_{l, l-1}} \sum_{k=1}^{l} \prod_{j=k}^{l} \frac{\Gamma_{j, j-1}}{\omega-\left(E_{j}-E_{j-1}\right)-\frac{i}{2}\left(\Gamma_{j, j-1}+\Gamma_{j-1, j-2}\right)}+c . c .,
$$

where $E_{j}$ is the energy of the state $|j\rangle$, and the spectrum is normalized as $\int S(\omega) d \omega=N$ by the quantity of photons emitted from $t=0$ to $t \rightarrow \infty$ if at the initial moment of time $t=0$ the occupied is the state $|N\rangle$. Below we consider several representative examples of usage of Eq. (1).

Generic three-level system. The spectrum can be presented in terms of the real and imaginary parts of two complex Lorentzians with the widths $\Gamma_{10}$ and $\Gamma_{21}+\Gamma_{10}$. The weights

\footnotetext{
*Corresponding author: amakarov@isan.troitsk.ru
} 
of the real parts are $1 \pm 2 \Gamma_{21} \Gamma_{10} /\left(4 \Delta^{2}+\Gamma_{21}^{2}\right)$, and the weights of the imaginary parts are $\mp 4 \Gamma_{10} \Delta /\left(4 \Delta^{2}+\Gamma_{21}^{2}\right)$, where $\Delta=2 E_{1}-E_{0}-E_{2}$ is the difference between the frequencies of $|1\rangle \rightarrow|0\rangle$ and $|2\rangle \rightarrow|1\rangle$ transitions. It can be noticed that at $\Delta=0$ and $\Gamma_{21}=2 \Gamma_{10}$ the spectrum becomes single-Lorentzian with the width $\Gamma_{10}$.

Harmonic oscillator. A generalization of the latter observation is the property of the system with $\Gamma_{j, j-1}=j \Gamma_{10}$, i.e. the harmonic oscillator. No matter whence the oscillator starts - its emission spectrum remains single-Lorentzian with the width $\Gamma_{10}$ of the lowest transition. This property is, of course, well known (see, e.g., Ref. [3]), but never has been derived using the consistent treatment in terms of the quantum electrodynamics.

Equidistant system with the equal decay rates $\Gamma$ for all transitions. Here, at the initial excitation of the $\mathrm{N}$-th level, the emission spectrum becomes narrower as the number $N$ grows (see the Figure). Finally, at $N \rightarrow \infty$ the spectrum (more precisely, the ratio $S=N$ ) degenerates into the $\delta$-function. This observation is in full correspondence with the spectrum of the elastic scattering in a week monochromatic laser field in terms of the quasienergy approach.

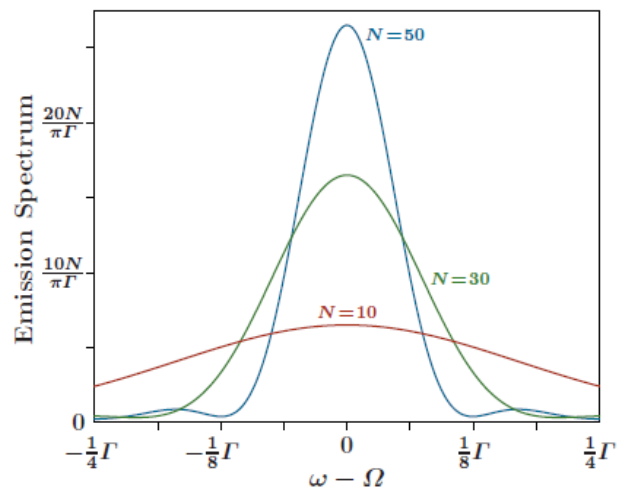

Fig. 1. Emission spectrum ( $\Omega$ is the frequency of all the successive transitions).

Dicke superradiance model. The Dicke model corresponds to a system of $N$ closely spaced non-interacting two-level atoms. At the initial excitation of all the atoms, a cascade of transitions occurs between the equidistant ( $\Omega$ is the energy difference) collective states (Dicke states). The emission spectrum calculated with the use of the Bethe Ansatz [4] coincides with that given by the general formula (1) where the transition rates are $\Gamma_{j, j-1}=j(N-j+1) \Gamma$. When $N \gg>1$, the spectrum takes the form

$$
S(\omega)=\frac{1}{2 \pi \Gamma} \frac{1}{\cosh ^{2}(\pi \varpi)}, \quad \varpi=\frac{\omega-\Omega}{N \Gamma}
$$

The work was supported by Russian Foundation for Basic Research (No. 15-02-05657).

\section{References}

1. V.B. Berestetskii, E.M. Lifshitz, L.P. Pitaevskii, Quantum Electrodynamics, Course of Theoretical Physics Vol. 4 (Pergamon, Oxford, 1982)

2. A.A. Makarov, V.I. Yudson, Phys. Rev. A 89, 053806 (2014)

3. B.Ya. Zeldovich, A.M. Perelomov, V.S. Popov, Sov. Phys. JETP 28, 308 (1969)

4. V.I. Rupasov, V.I. Yudson, Sov. Phys. JETP 60, 927 (1984) 